\title{
VIEWPOINT
}

\section{Should Evidence Come with an Expiration Date?}

\author{
Palmer Greene, $B A^{1,2}$, Vinay Prasad, $M D, M P H^{3}$, and Adam Cifu, $M D^{7}$ \\ 'University of Chicago, Chicago, IL, USA; ${ }^{2}$ Division of Hematology and Medical Oncology, Knight Cancer Institute, Oregon Health and Science \\ University, Portland, OR, USA; ${ }^{3}$ Department of Public Health and Preventive Medicine, Oregon Health and Science University, Portland, OR, USA. \\ J Gen Intern Med 34(7):1356-7 \\ DOI: $10.1007 / \mathrm{s} 11606-019-05032-4$ \\ (c) Society of General Internal Medicine 2019

\section{WHY DOES A THERAPY PROVEN EFFECTIVE STOP WORKING?}

I is not unusual for accepted therapies to be abandoned in the face of new evidence. Usually, this occurs as a medical reversal, when a therapy adopted without strong evidence is later shown to be ineffective in a well-designed randomized trial. Sometimes, however, therapies once supported by robust evidence are proven to no longer work. The basis of this declining efficacy is diverse but includes changing population risk, newly adopted adjunctive medical therapy, and, for screening interventions, more effective treatments, which obviate the gains from early detection. Recently published trials showing aspirin's lack of efficacy in the primary prevention of cardiovascular disease (CVD) offer an example of this phenomenon. The lesson of aspirin for primary prevention is a broad one. What was once well-supported by data may prove ineffective in the future. Conversely, some interventions found to be ineffective may have worked if tested a decade earlier. We propose an "evidentiary statute of limitations" as a core principle of evidence-based medicine, wherein consideration is given to factors that may require the efficacy of accepted therapies to be reevaluated.

Multiple trials have evaluated the efficacy of aspirin in the primary prevention of CVD, and recent studies find that the benefits are outweighed by the harms. ${ }^{1}$ A representative study by Gaziano and colleagues randomized participants at moderate risk for cardiovascular events to aspirin or placebo. ${ }^{1}$ The primary endpoint was a composite of cardiovascular events, and adverse effects included hemorrhagic events. In the intention-to-treat analysis, there was no benefit to aspirin therapy (hazard ratio [HR] $0.96 ; 95 \%$ CI $0.81-1.13 ; p=$ $0.6038)$. Gastrointestinal bleeding events were more common in the aspirin group (HR 2.11; 95\% CI 1.36-3.28; $p=0.0007$ ).

This contrasts recent studies with those from decades ago when aspirin was shown to be effective. The Physicians' Health Study, one of the early successes in the era of evidence-based medicine, and subsequent meta-analysis demonstrated efficacy. ${ }^{2,3}$

Received December 12, 2018

Revised January 2, 2019

Accepted April 4, 2019

Published online May 6, 2019
The physiologic effects of aspirin are no different today than they were 20 years ago. Nor is this an example of regression to the mean as multiple older studies showed similar levels of efficacy for aspirin. The decreasing efficacy of aspirin is likely due to a change in the characteristics of the population (or population studied). Compared with the 1980s, participants in the current studies have healthier lifestyles and improved risk factor management. Additionally, the participants enrolled in recent studies include a mix of men and women while older studies were overwhelmingly male. Although difficult to compare studies of different population done decades apart, the population differences are reflected in the rate of the composite endpoint that was $4.48 \%$ in the placebo group of the Gaziano study: a rate lower than all but one of the studies included in the meta-analysis referenced above (and markedly lower than that predicted by the American College of Cardiology/American Heart Association risk calculator). ${ }^{1}$ The rate of myocardial infarction (MI) in this trial was about 373 per 100,000 person-years compared with 439.7 in the Physicians' Health Study. 1, 2

Aspirin's waning efficacy is not an isolated example. Other therapies whose benefit was proven have been found to be ineffective in later studies. Trials initially showed a benefit of angiotensin-converting enzyme inhibitor (ACE-I) therapy in patients with vascular disease. ${ }^{4}$ A later trial, conducted in patients on more up-to-date preventative therapy (higher rates of lipid-lowering therapy and aspirin use) no longer revealed a benefit. 5

It is also possible to identify negative trials that might have been positive had they been performed at an earlier date. A 2009 trial that examined the benefit of adenosine-stress radionuclide myocardial perfusion imaging to screen for coronary artery disease (CAD) in patients with type 2 diabetes found no improvement in cardiac death or non-fatal MI. ${ }^{6}$ The negative results of this trial were, to a great extent, based on the lowerthan-expected event rates in the participants. Had this trial been conducted 10 years earlier when cardiac event rates would have been higher (and closer to those on which the power calculations were based), it is possible that CAD screening would have been written into guidelines and still be in use today.

There are interventions accepted today that one might anticipate will not be effective in the future. Screening for lung 
cancer has already been shown to result in rates of overdiagnosis that may be unacceptable given the relatively small benefit. $^{7}$ As the intensity of smoking decreases and the success of therapies for lung cancer increases, it is possible that, even among patients who qualify, screening will no longer be beneficial. Screening for abdominal aortic aneurysms may follow a similar trajectory.

Should the evidence on which we base our practice come with an expiration date? An "evidentiary statute of limitations" would require the occasional reassessment of accepted therapies to consider which might no longer be of use-possibly because of changes in the population as a whole, a changing understanding of whom the treatment is appropriate for, or evolving therapies for the prevention or treatment of the disease in question. Not only should we consider if older data still applies, we should also strive to anticipate the factors to which the results of a newly published positive study might be sensitive. For instance, is there an event rate in the control group below which the harms of the therapy might outweigh the benefit? Is there a treatment success rate that, when achieved, would make screening inefficient?

A cyclic re-assessment of clinical data is a necessary next step for evidence-based medicine. Only by periodically checking whether our interventions are still working may we confidently treat our patients in a manner built on a solid foundation of reliable and up-to-date evidence.

Corresponding Author: Adam Cifu, MD; University of Chicago, Chicago, IL, USA (e-mail: adamcifu@uchicago.edu).

\section{Compliance with Ethical Standards:}

Conflict of Interest: Dr. Prasad reports receiving royalties from his book Ending Medical Reversal; that his work is funded by the Laura and John Arnold Foundation; that he has received honoraria for Grand Rounds/lectures from several universities, medical centers, and professional societies and payments for contributions to Medscape. Dr. Cifu reports receiving royalties from his books Ending Medical Reversal and Symptom to Diagnosis: An Evidence Based Guide. All remaining authors declare that they do not have a conflict of interest.

\section{REFERENCES}

1. Gaziano JM, Brotons C, Coppolecchia R, et al. Use of aspirin to reduce risk of initial vascular events in patients at moderate risk of cardiovascular disease (ARRIVE): a randomised, double-blind, placebo-controlled trial. Lancet 2018;392(10152):1036-1046.

2. Steering Committee of the Physicians' Health Study Research Group. Final report on the aspirin component of the ongoing Physicians' Health Study. N Engl J Med. 1989;32 1(3):129-35.

3. Sanmuganathan PS, Ghahramani P, Jackson PR, et al. Aspirin for primary prevention of coronary heart disease: safety and absolute benefit related to coronary risk derived from meta-analysis of randomised trials. Heart 2001; 85 :265-271

4. Effects of an Angiotensin-Converting-Enzyme Inhibitor, Ramipril, on Cardiovascular Events in High-Risk Patients. N Engl J Med. 2000;342(3):145-153.

5. Angiotensin-Converting-Enzyme Inhibition in Stable Coronary Artery Disease. N Engl J Med. 2004;351(20):2058-2068.

6. Young LH, Wackers FJT, Chyun DA, et al. Cardiac Outcomes After Screening for Asymptomatic Coronary Artery Disease in Patients With Type 2 Diabetes. JAMA. 2009;301(15): 1547.

7. Heleno B, Siersma V, Brodersen J. Estimation of Overdiagnosis of Lung Cancer in Low-Dose Computed Tomography Screening. JAMA Intern Med. 2018; 178(10):1420

Publisher's Note Springer Nature remains neutral with regard to jurisdictional claims in published maps and institutional affiliations. 